\title{
Moths Diversity of Ziro in Lower Subansiri District, Arunachal Pradesh India
}

\author{
Mrunalini Sonne $^{1}$, Shreyas Gaikwad ${ }^{2}$ \\ ${ }^{1}$ Annasaheb Kulkarni Department of Biodiversity, M.E.S. Abasaheb Garware College, Pune-411004, Maharashtra, India. \\ ${ }^{2}$ B3/102 Pride Regency Viman nagar, Pune - 411014, Maharashtra, India. \\ DOI: 10.29322/IJSRP.11.07.2021.p11535 \\ http://dx.doi.org/10.29322/IJSRP.11.07.2021.p11535
}

\begin{abstract}
Moths were researched in the Lower Subansiri district of Arunachal Pradesh, India. A preliminary field opportunistic survey will be conducted in Old Ziro, Lower Subansiri district, Arunachal Pradesh, during September-October 2020 to document the common moth, in total 38 moth species were observed foraging in day-night. With the help of photo documentation listed with taxa and photographs. After the present study, the moth fauna of Ziro comprises a total of 40 species belonging to 12 families. Of these, species richness of family Geometridae was found to be, Noctuidae, Erebidae, Crambidae, Sphingidae, Arctiidae, Uraniidae, Drepanidae, Saturniidae, Bombycidae, Lasiocampidae, Cossidae.
\end{abstract}

Index Terms- Arunachal Pradesh, Lower Subansiri, Ziro, Moth

\section{INTRODUCTION}

A total of 1,27,000 moth species have been identified around the world (Alfred et al.). According to a previous study, India has about 12,000 species (Chandra and Nema 2007). Bees and wasps pollinated $56 \%$ plant species, butterflies and moths were $11 \%$, flies $10 \%$, beetles $3 \%$, and birds $12 \%$, while wind pollinated
8\% (Wyckhyus, 2019). Therefore, as an initiative before a detailed study, present observation of moths of Arunachal Pradesh was focused to document in certain areas of the state to plan the future study and helpful to global lepidoptera checklist assessments.

\section{MATERIALS AND METHODS}

Study area: Old Ziro is a town in Arunachal Pradesh. It is the district headquarters of the Lower Subansiri district and one of the state's oldest cities. At an elevation of 1688 meters, the settlement is located at $27.63^{\prime} \mathrm{N} 93.83^{\prime} \mathrm{E}$. The moth checklist offered in this paper is based on an opportunistic survey that we conducted primarily in old Ziro during nights over a period of two month in 2020, during the months of September and October. Moths were not collected but were primarily identified using digital colour photographs. Old Ziro were surveyed primarily at night using a $160 \mathrm{~W}$ mercury vapour bulb, although there are several records from random sampling during the day from the same locations. The study sites are shown in Fig. 1. 
Figure 1: Study Area

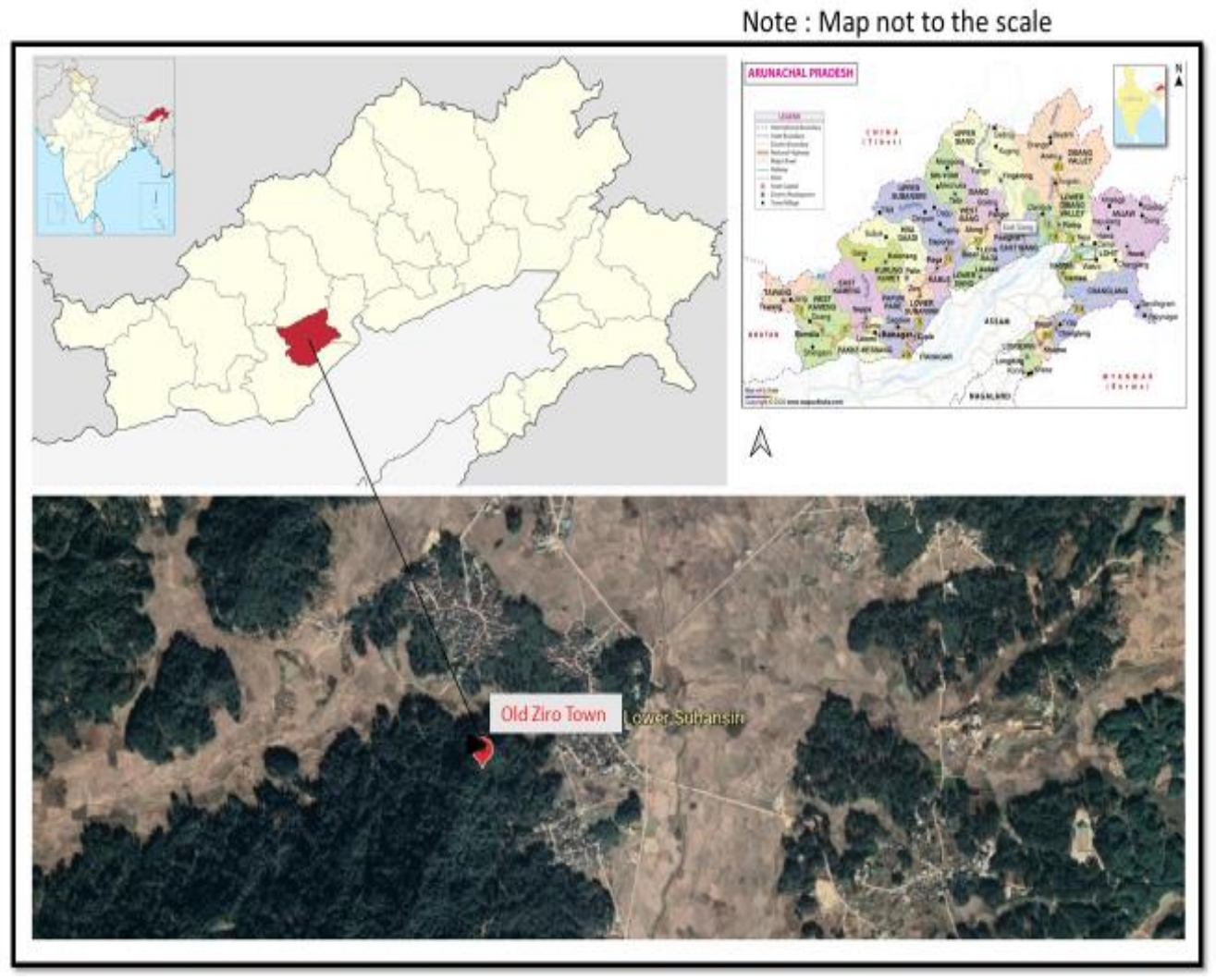

A Canon EOS 700D with a 55-200mm f/4-5.6 VR DX lens was used to shoot moths in the field. The Arunachal Pradesh Forest Department issued permission for photographing naturally dead or alive moths. On (https://www.gbif.org), (https://www.indiabiodiversity.org),

(https://www.britannica.com), the available information was used to identify and classify the moths, literature Moore (1840-80), Hampson (1891-96), Bell and Scott (1937), Holloway (19832011), Pinratana (1990-2007), Kirti and Singh (2002-2006), Chandra, K and Sam bath (2013).

\section{RESULTS AND DISCUSSION}

The study revealed that a total of 102 species belong to 81 genera, 24 subfamilies, 12 families under seven super families. Arora and Chaudhury researched the moth fauna of Arunachal Pradesh's Arctiidae family (1982) Kirti et al. (2005) inventoried 105 species of the family Arctiidae from north-eastern India. Chandra, K and Sam bath (2013) inventoried 102 species Moth diversity of Tawang District, Arunachal Pradesh, India. But, the information on moth fauna of Tawang District has not been fully studied and District wise Moth diversity of Lower Subansiri, Arunachal Pradesh, India species are reported. 
Figure 2: Family compositions of moth

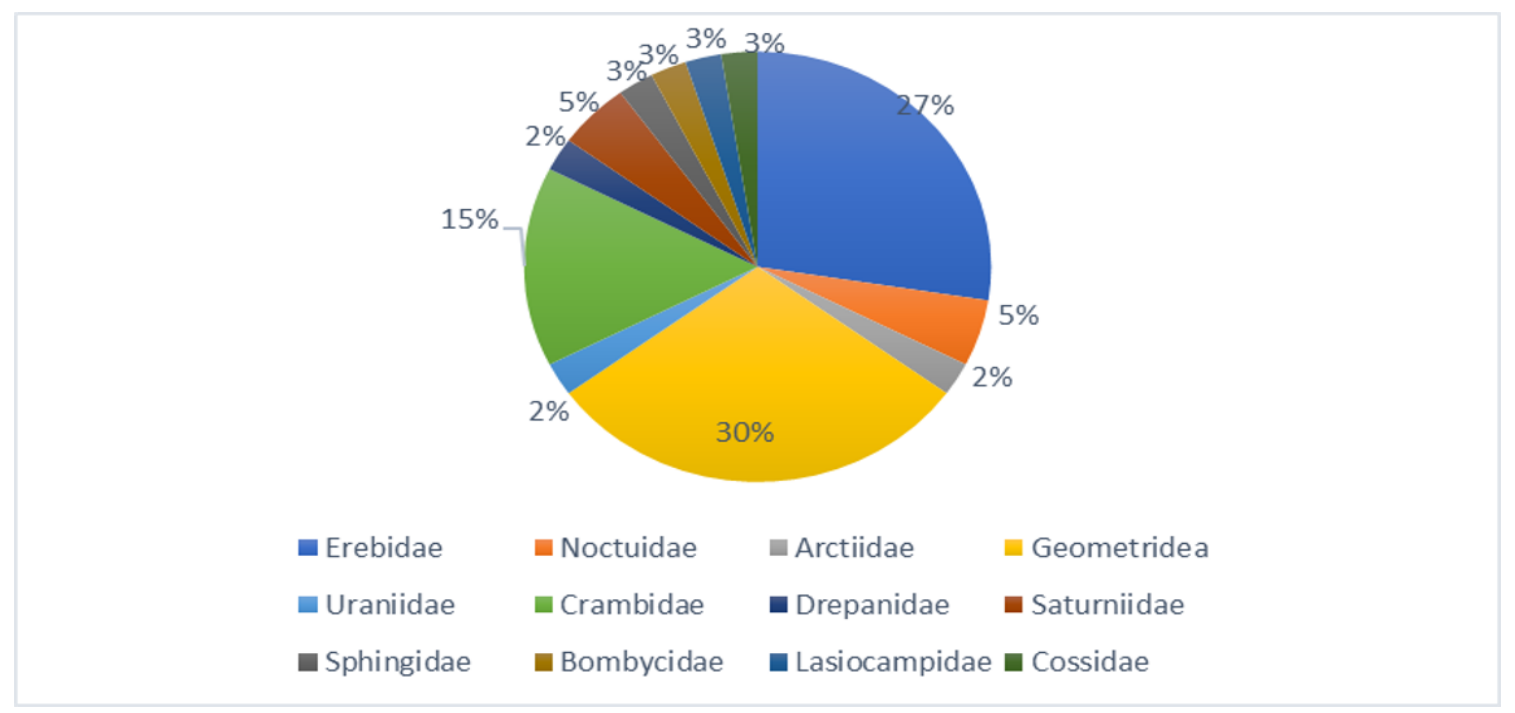

In this investigation, we photographed 40 species and recognised 39 genera under 12 families (Table 1). A The Geometridae family dominated with $30 \%$ of the total species observed, followed by the families, Erebidae (27\%), Crambidae
(16\%), Noctuidae (5\%), Saturniidae (5\%), Sphingidae (3\%), Drepanidae (3\%), Bombycidae (3\%), Lasiocampidae (3\%), Cossidae (3\%) Arctiidae (2\%), Uraniidae (2\%).

Table 1: The recorded Moths in the study area.

\begin{tabular}{|c|c|c|c|c|c|}
\hline Sr.No & Family & Sub-family & Scientific Name & Autor/Year & Common Name \\
\hline 1 & Erebidae & Erebinae & Lygniodes hypoleuca & Guenée, 1852 & - \\
\hline 2 & Erebidae & Arctiinae & Cyana sp. & Walker , 1854 & - \\
\hline 3 & Erebidae & Erebinae & Aglaomorpha plagiata & Walker 1855 & Yellow-banded Tiger \\
\hline 4 & Erebidae & Erebinae & Bastilla joviana & Cramer, 1782 & _- \\
\hline 5 & Erebidae & Arctiinae & Nyctemera adversata & Schaller, 1788 & Marbled White Moth \\
\hline 6 & Erebidae & Erebinae & Lygniodes schoenbergi & Pagenstecher, 1890 & - \\
\hline 7 & Erebidae & Erebinae & Erebus macrops & Linnaeus, 1768 & Common Owl Moth \\
\hline 8 & Erebidae & Erebinae & Mocis proverai & Zilli, 2000 & Sugarcane Looper \\
\hline 9 & Erebidae & Lymantriinae & Pida patrana & Moore, 1859 & - \\
\hline 10 & Erebidae & _ & Phyllodes sp. & Philippi, 1841 & - \\
\hline 11 & Erebidae & - & Hemeroplanis sp. & Smith, 1893 & - \\
\hline 12 & Noctuidae & _- & Thyas juno & Dalman, 1823 & Fruit-piercing moth \\
\hline 13 & Noctuidae & _- & Singara diversalis & Walker , 1865 & - \\
\hline 14 & Arctiidae & Arctiinae & Lemyra rhodophilodes & Hampson, 1909 & - \\
\hline 15 & Crambidae & Pyraustinae & Udea rubigalis & Guenee, 1854 & Celery Leaftier moth \\
\hline 16 & Crambidae & Spilomelinae & Conogethes punctiferalis & Guenee, 1854 & Castor capsule borer \\
\hline 17 & Crambidae & Spilomelinae & Meroctena tullalis & Walker, 1859 & - \\
\hline 18 & Crambidae & Spilomelinae & Palpita vitrealis & Rossi, 1794 & Jasmine moth \\
\hline 19 & Crambidae & Spilomelinae & Hymenia perspectalis & Hubner ,1796 & Beet webworm \\
\hline 20 & Crambidae & Spilomelinae & Nevrina procopia & Stoll, 1781 & Wheel Moth \\
\hline 21 & Geometridea & Geometridae & Eumelea rosalia & Stoll, 1781 & - \\
\hline 22 & Geometridae & Larentiinae & Entephria flavicinctata & Hübner, 1813 & Yellow-banded Tiger \\
\hline 23 & Geometridae & Sterrbinae & Pleuroprucha insulsaria & Guenée, 1858 & Common tan wave \\
\hline 24 & Geometridae & Ennominae & Cleora alienaria & Walker, 1860 & - \\
\hline 25 & Geometridae & _- & Chiasmia emersaria & Walker 1861 & Chiasmia emersaria \\
\hline
\end{tabular}




\begin{tabular}{|l|l|l|l|l|l|}
\hline 26 & Geometridae & Geometrinae & Maxates sp. & Moore, 1887 & Geometrid moth \\
\hline 27 & Geometridae & Ennominae & Antipercnia cordiforma & Noue, 1978 & - \\
\hline 28 & Geometridea & - & $\begin{array}{l}\text { Thinopteryx } \\
\text { crocoptera assamensis }\end{array}$ & Swinhoe, 1916 & $\begin{array}{l}\text { Yellow Butterfly } \\
\text { Moth }\end{array}$ \\
\hline 29 & Geometridae & - & Cyclophora obstataria & Walker, 1861 & - \\
\hline 30 & Geometridae & Ennominae & Erebomorpha fulgurita & Walker, 1860 & - \\
\hline 31 & Geometridae & - & Ourapteryx pallidula & Inoue, 1985 & - \\
\hline 32 & Geometridae & Ennominae & Abraxas sp. & Leach, 1815 & $\begin{array}{l}\text { common magpie } \\
\text { moth }\end{array}$ \\
\hline 33 & Uraniidae & Uraniinae & Lyssa zampa, & Butler, 1869. & - \\
\hline 34 & Drepanidae & Drepaninae & Teldenia specca & Wilkinson, 1967 & Hooktip moth \\
\hline 35 & Saturniidae & Saturniinae & Actias selene & Hübner, 1806 & Indian moon moth \\
\hline 36 & Saturniidae & Antheraea & Antheraea pernyi & $\begin{array}{l}\text { Guerin-Meneville, } \\
1855\end{array}$ & $\begin{array}{l}\text { Chinese oak silk } \\
\text { moth }\end{array}$ \\
\hline 37 & Sphingidae & Spilomelinae & Theretra latreillii lucasii & Walker, 1856 & $\begin{array}{l}\text { Pale brown hawk } \\
\text { mouth }\end{array}$ \\
\hline 38 & Bombycidae & Sphingidae & Theretra nessus & Drury, 1773 & Yam Hawk moth \\
\hline 39 & Lasiocampidae & - & Argonestis flammans & $\begin{array}{l}\text { George Hampson } \\
1893\end{array}$ & - \\
\hline 40 & Cossidae & - & Zeuzera pyrina & Linnaeus, 1761). & Wood leopard moth \\
\hline
\end{tabular}

Arunachal Pradesh is full of flora and fauna which has to strike a balance between natural biodiversity and climatic changes which is challenging. This paper is providing a row sketch regarding the species diversity of moth in Ziro valley; it may be helpful for finding out moth pollination networks in the nearest places along with their native host plant relationship. In India few or not a signal study addressing ecological questions behind the diversity and distribution pattern of moth assemblages. The diversity of moth fauna as recorded by the earlier studies from different parts of Arunachal Pradesh showed a wide variation. All the work has been addressing the taxonomy of moth with or without photograph; still the inventory of these taxa for a single town, district and state wise is not complete. 

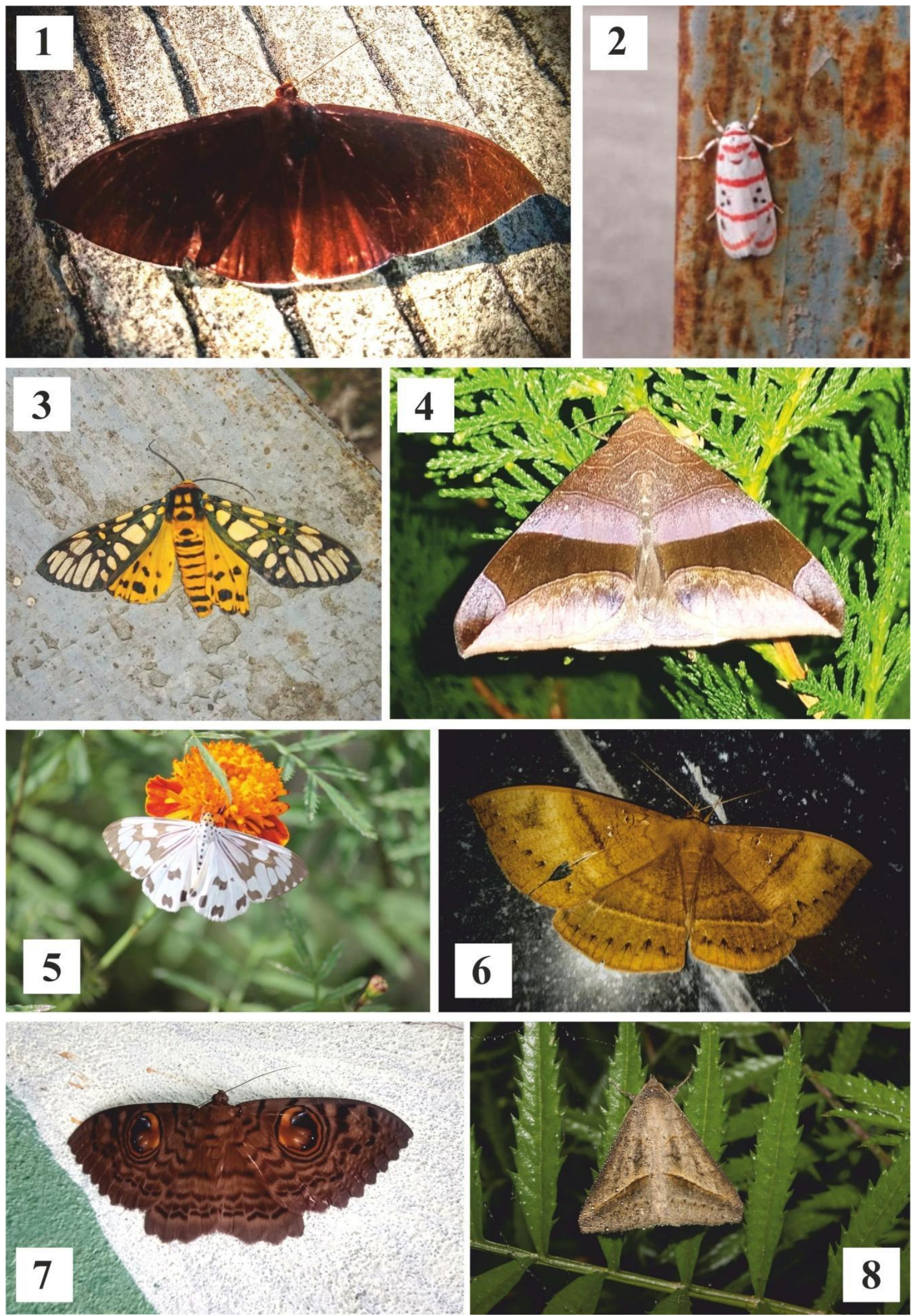

(1) Lygniodes hypoleuca, (2) Cyana sp., (3) Aglaomorpha plagiata, (4) Bastilla joviana, (5) Nyctemera adversata, (6) Lygniodes schoenbergi, (7) Erebus macrops, (8) Mocis proverai 

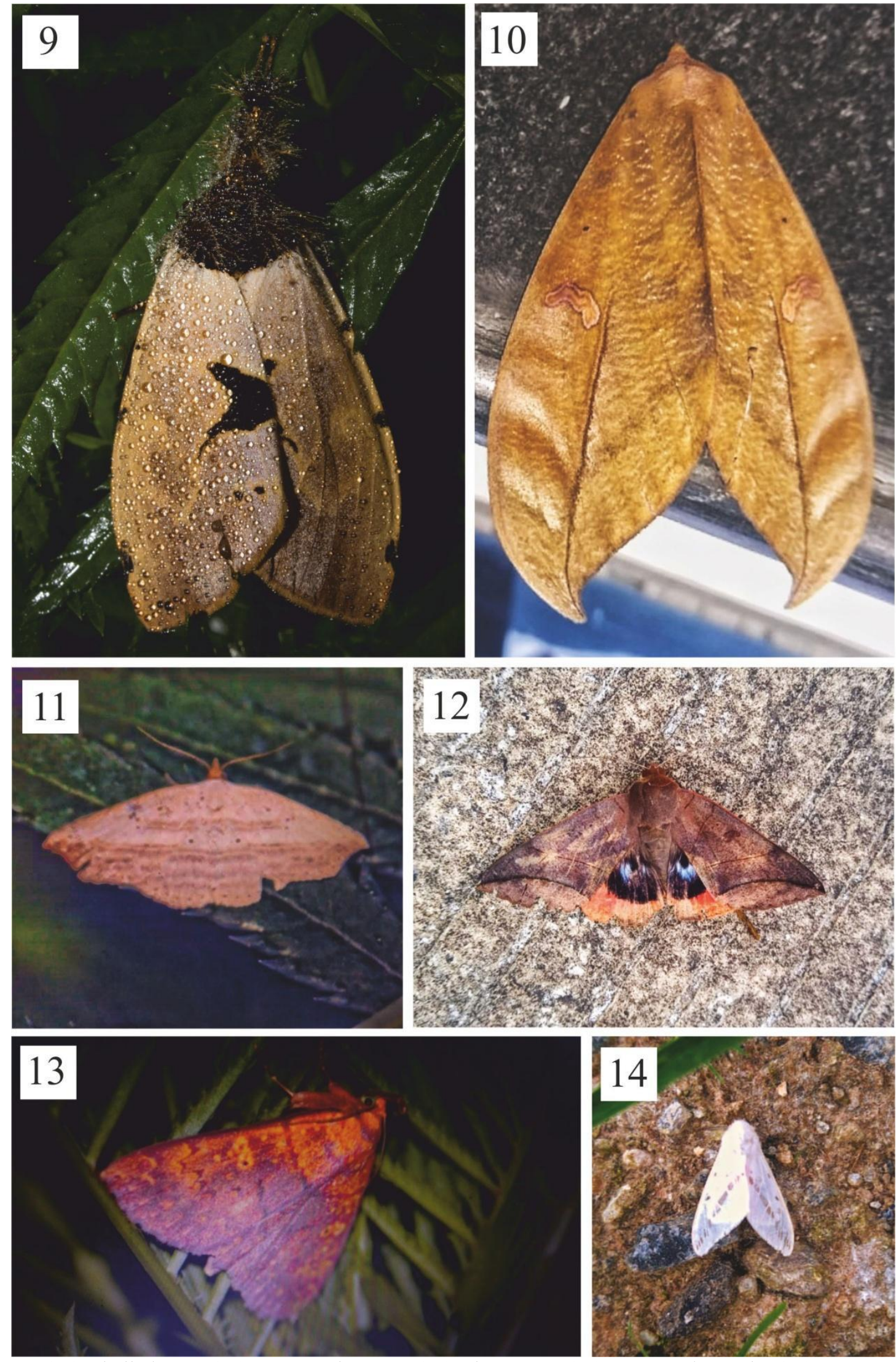

(9) Pida patrana, (10) Phyllodes sp., (11) Hemeroplanis sp., (12) Thyas juno, (13) Singara diversalis, (14) Lemyra rhodophilodes 

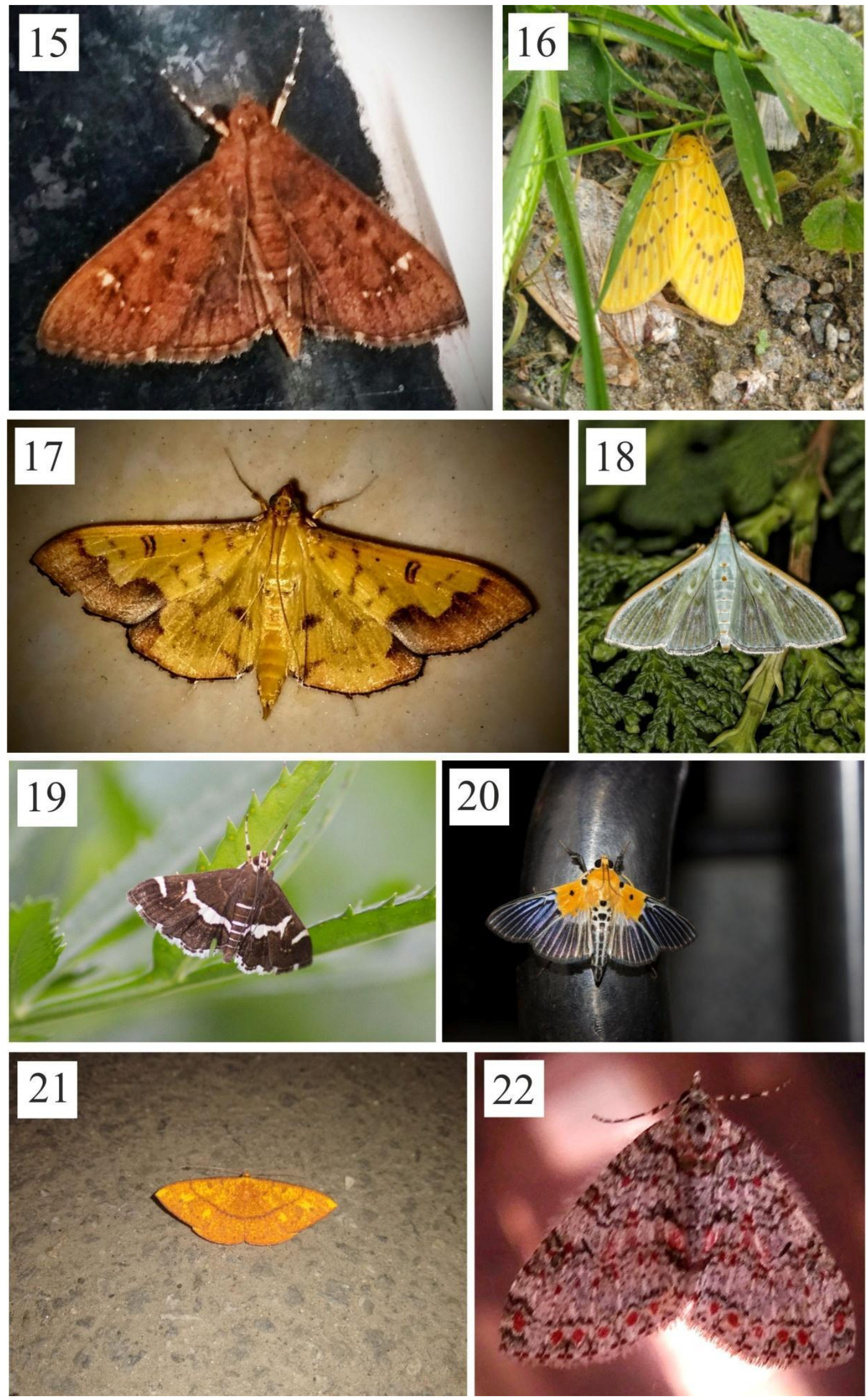

(15) Udea rubigalis, (16) Conogethes punctiferalis, (17) Meroctena tullalis, (18) Palpita vitrealis, (19) Hymenia perspectalis, (20) Nevrina procopia, (21) Eumelea rosalia, (22) Entephria flavicinctata 

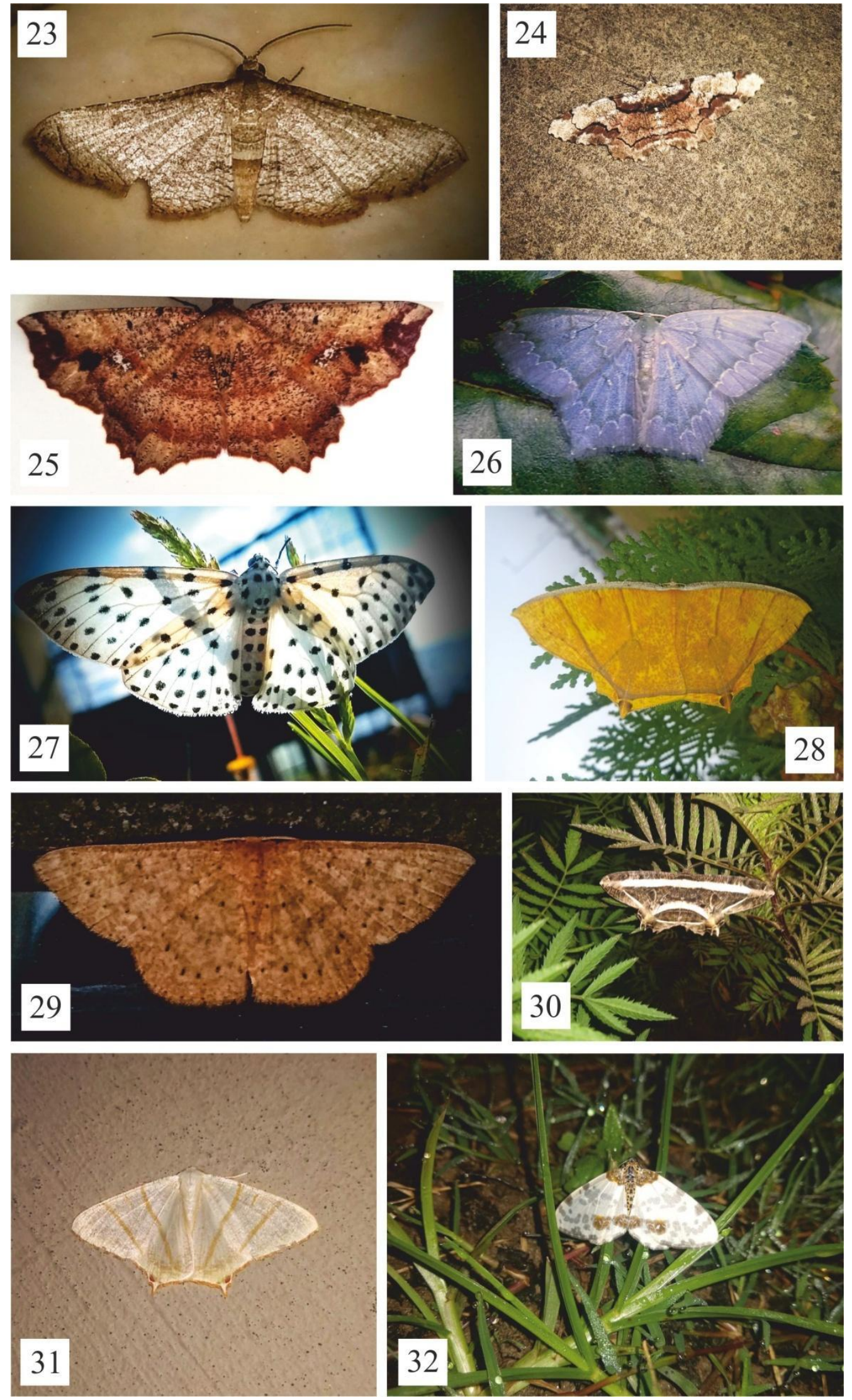

(23) Pleuroprucha insulsaria, (24) Cleora alienaria, (25) Chiasmia emersaria, (26) Maxates sp., (27) Antipercnia cordiform, (28) Thinopteryx crocoptera assamensis, (29) Cyclophora obstataria, (30) Erebomorpha fulgurita, (31) Ourapteryx pallidula, (32) Abraxas sp. 

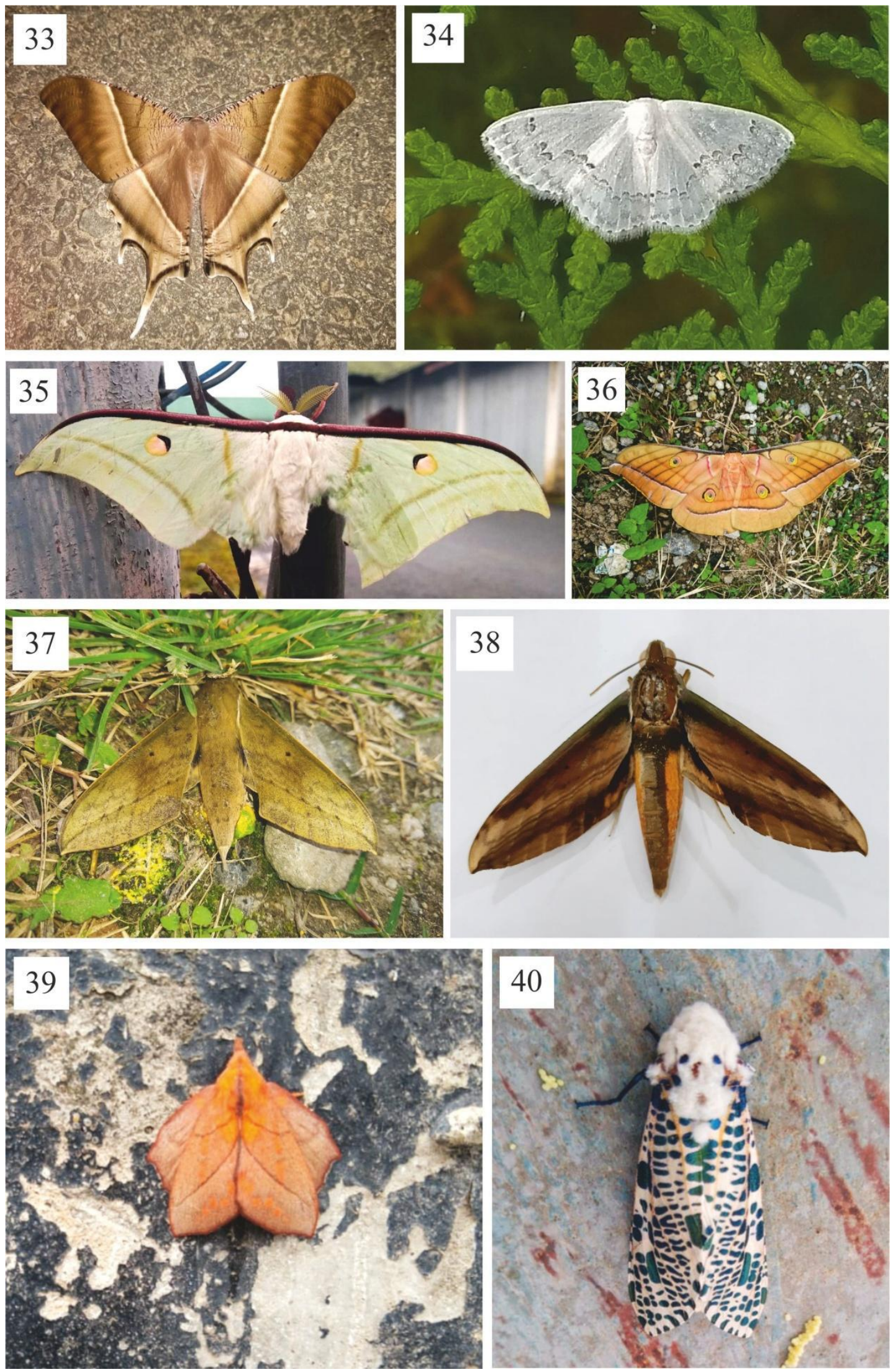

(33) Lyssa zampa, (34) Teldenia specca, (35) Actias selene, (36) Antheraea pernyi, (37) Theretra latreillii lucasii, (38) Theretra Nessus, (39) Argonestis flammans, (40) Zeuzera pyrina. 


\section{ACKNOWLEDGEMENTS}

The authors are grateful to Shri Abhinav Kumar, Div. Forest Officer, Ziro Arunachal Pradesh for permeation granted to photography. Authors also extend they're thanks to Sneha Shahane and Prasad Gond for helping in moth identification.

\section{REFERENCES}

[1] Arora, 0.8. and Chaudhary, M. 1982. On the Lepidopterous fauna of Arunachal Pradesh and adjoining areas of Assam in North-East India. Family Arctiidae. Zoological Survey of India, Technical Mongraph.

[2] Alfred, J.R.B., A.K. Das and A.K. Sanyal (1998). Faunal Diversity in India. ENVIS Centre Zoological Survey of India, Kolkata

[3] Beccaloni, G.W., M.J., Scoble, G.S. Robinson, A.C. Downton and S.M. Lucas (2003). Lepindex. Natural history museum London.

[4] Bell, T.R.D. \& F.B. Scott (1937). The Fauna of British India including Ceylon and Burma Taylor and Francis, London.

[5] Butler, A.G. (1881). Elcysma translucida. Transactions of the Entomological Society of London.
[6] Chandra, K and Sam bath, S. (2013). Moth diversity of Tawang District, Arunachal Pradesh, India. Journal of Threatened Taxa, 3565-3570.

[7] Hampson, G.F. (1895). The Fauna of British India including Ceylon and Burma, London.

[8] Hampson, G.F. (1896). The Fauna of British India including Ceylon and Burma Taylor and Francis, London.

[9] Kirti, and Sodhi, (2002). Arctiid diversity in Himachal Pradesh (Arctiidae: Lepidoptera). Geobios.

[10] Kirti, J.S. and Sodhi, (2002). Studies on footman moths of Sikkim (Arctiidae: Lithosiinae: Lepidoptera). Journal Hill Research.

\section{AUTHORS}

First Author - Mrunalini Sonne, Annasaheb Kulkarni Department of Biodiversity, M.E.S. Abasaheb Garware College, Pune-411004, Maharashtra, India.

Second Author - Shreyas Gaikwad, B3/102 Pride Regency Viman nagar, Pune - 411014, Maharashtra, India., Corresponding author email: ssonne929@gmail.com 